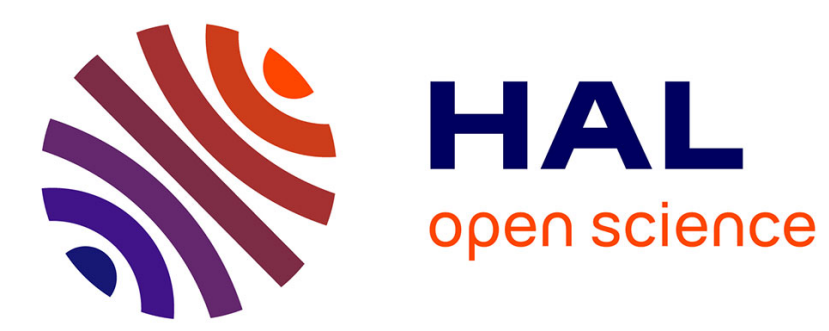

\title{
Décider la mort et prélever les organes : la question de l'extension des conditions du prélèvement d'organes
}

Caroline Guibet Lafaye, Louis Puybasset

\section{To cite this version:}

Caroline Guibet Lafaye, Louis Puybasset. Décider la mort et prélever les organes: la question de l'extension des conditions du prélèvement d'organes. Éthique publique - revue internationale d'éthique sociétale et gouvernementale , 2010, 12 (2), p. hal-00496595

\section{HAL Id: hal-00496595 \\ https://hal.science/hal-00496595}

Submitted on 25 May 2017

HAL is a multi-disciplinary open access archive for the deposit and dissemination of scientific research documents, whether they are published or not. The documents may come from teaching and research institutions in France or abroad, or from public or private research centers.
L'archive ouverte pluridisciplinaire HAL, est destinée au dépôt et à la diffusion de documents scientifiques de niveau recherche, publiés ou non, émanant des établissements d'enseignement et de recherche français ou étrangers, des laboratoires publics ou privés. 


\title{
Décider la mort et prélever les organes : la question de l'extension des conditions du prélèvement d'organes
}

\author{
Caroline Guibet Lafaye et Louis Puybasset
}

\section{Résumé :}

La référence à la pénurie d'organes est un lieu commun du discours politique et social associé au don. Dans ce contexte, se pose la question d'une extension possible des conditions d'inclusion des patients parmi les donneurs d'organes. Nous analyserons ici, dans le cas spécifique des patients pour lesquels un arrêt des thérapeutiques est décidé, les conséquences morales et sociales ainsi que les dilemmes moraux suscités par une telle extension. Nous montrerons notamment que l'indétermination des normes de référence autorise des pratiques divergentes et moralement contestées au sein des pays occidentaux. Il s'agira également de souligner les formes d'aménagement normatif induites par cette évolution de la pratique médicale.

Mots-clés : Prélèvement d'organes, mort, normes, consentement, utilitarisme.

Abstract: Social and political discourse still emphasizes the "shortage" of transplantable organs. In this international and national context, broadening the pool of donors is at stake. Addressing the specific case of withdrawing life-sustaining treatments, the paper will analyse the social and moral consequences of this extension and the moral dilemmas that it creates. We will also underline how the indeterminacy of fundamental norms gives rise to divergent and morally controversial practices in Western countries. Finally we will highlight the kind of normative evolution prompted by such new medical practices.

Key words : Organ donation, death, norms, consent, utilitarianism.

La question et la réflexion sur le prélèvement d'organes s'inscrivent aujourd'hui sous le paradigme de la pénurie. Le prélèvement d'organes chez les patients décédés après arrêt cardiaque (DDAG) est autorisé aujourd'hui, en France, en référence au cadre défini par la classification de Maastricht, établie en 1995 lors d'une Conférence internationale de consensus. Cette classification distingue les personnes ayant fait un arrêt cardiaque en 
présence (classe II) ou non (classe I) de secours qualifiés ${ }^{1}$, les personnes pour lesquelles la mort survient suite à une décision d'arrêt de traitements en réanimation (classe III) et enfin les personnes chez qui survient un arrêt cardiaque inopiné, lors de la réanimation d'un donneur en mort encéphalique (classe IV). En France, seuls sont autorisés actuellement les prélèvements de reins et de foie chez les personnes des catégories I, II et IV.

En référence à ce contexte dit de pénurie, se pose la question d'une extension des conditions du prélèvement d'organes aux patients de la catégorie III de la classification de Maastricht (DDAC III). Elle se pose d'autant plus qu'en France, en particulier pour les patients inconscients et gravement cérébrolésés aussi bien que pour les patients conscients demandant l'arrêt des thérapeutiques, aucune interdiction légale de prélèvement n'a été prise $^{2}$. Seul existe un «moratoire » de l'Agence de Biomédecine exprimé dans son protocole du mois de mars 2007, établi conformément à l'article R1232-4-1 $2^{\text {ème }}$ alinéa du Décret 2005949 du 2 août 2005 sur le prélèvement d'organes sur donneur à cœur arrêté3.

Or l'évolution du patient vers la mort dépendant, dans le cas de ces patients, d'une décision et d'un processus intentionnel, le prélèvement d'organes pose ici des problèmes éthiques spécifiques. Dans ce contexte, pourrait donc s’initier une évolution des normes ayant des incidences sociales majeures.

Dans ce qui suit, notre propos ne consistera pas à s'opposer à l'extension du prélèvement d'organes aux patients DDAC III, en convoquant l'argument de la «pente glissante » pour suggérer par exemple que si l'on accepte le prélèvement pour ce type de patients, on en viendra à accepter, inévitablement, le prélèvement de patients faiblement autonomes ou polydéficients par exemple. Il s'agira plutôt de montrer l'incidence de l'indéterminabilité des normes sur les pratiques et les coûts qu'elle induit, en termes éthiques, c'est-à-dire en termes de respect de principes éthiques fondamentaux et de convictions morales individuelles. Nous verrons en effet dans un premier temps que les pratiques actuelles, en matière de prélèvements d'organes, révèlent des effets et des phénomènes d'autorisation, induits par les termes et les

\footnotetext{
${ }^{1}$ La classe I concerne : «Les personnes qui font un arrêt cardiaque en dehors de tout contexte de prise en charge médicalisée et pour lesquelles le prélèvement d’organes ne pourra être envisagé que si la mise en œuvre de gestes de réanimation de qualité a été réalisée moins de trente minutes après l'arrêt cardiaque ». La classe II concerne : «Les personnes qui font un arrêt cardiaque en présence de secours qualifiés, aptes à réaliser un massage cardiaque et une ventilation mécanique efficaces, mais dont la réanimation ne permettra pas une récupération hémodynamique » (« unsuccesful rescussitation »).

${ }^{2}$ Contrairement à la procédure légale très stricte qui encadre les prélèvements d'organes des patients en état de mort encéphalique (décret n96-1041 du 2 décembre 1996 relatif au constat de la mort préalable au prélèvement d'organes, de tissus et de cellules à des fins thérapeutiques ou scientifiques), l'état de mort encéphalique consécutif à l'arrêt cardiaque, dans le cadre des prélèvement d'organes de patient décédé à cœur arrêté n’a pas été documenté dans la législation française.

${ }^{3}$ Voir http://www.agence-biomedecine.fr/presse/rappel-sur-le-protocole-en-vue-d-un-prelevement-apres-arretcardiaque-1.html (visité le 14/12/09).
} 
critères choisis pour la description d'états décisifs, en l'occurrence la description de la mort, la notion d'irréversibilité, l'agonie et la fin de vie. Les pratiques de prélèvement s'adossent à une dimension performative, liée à la définition des critères de la mort. La description « des états du monde » joue ici un rôle fondamental et a une incidence décisive sur les pratiques dont témoigne, comme nous le verrons, le fait que les soignants doutent parfois eux-mêmes que l'état de mort soit atteint par le patient (D'Alessandro et al., 2008, p. 1079).

Nous nous demanderons ensuite dans quelle mesure la demande sociale - visant à obtenir davantage de greffons - est à même de justifier les prélèvements d'organes sur les patients de la classe III de Maastricht. Nous viserons à cerner la légitimité des usages qui peuvent être fait du corps dans le cas considéré, c'est-à-dire du corps mourant. Enfin nous tenterons de déterminer, en la matière, jusqu'à quel point on se trouve fondé, d'un point de vue moral ou politique, à réagir à des situations nouvelles par des réaménagements normatifs, c'est-à-dire par l'adoption de nouvelles normes ou de nouvelles interprétations des normes acceptées (comme la règle du «donneur mort») ou par des réaménagements dans les valeurs de référence, encadrant l'agir institutionnalisé.

\section{Indéterminabilité des normes et critères de référence}

\subsection{Pratiques existantes et patients concernés}

Le prélèvement d'organes sur patients relevant de la classe III de Maastricht, c'est-à-dire « les personnes pour lesquelles une décision d'un arrêt de traitements en réanimation est prise en raison de leur pronostic », se trouve déjà réalisé dans une partie du monde occidental, en particulier dans certains États des États-Unis, en Grande-Bretagne, aux Pays-Bas et en Belgique. Le premier protocole concernant les prélèvements d'organes sur patients décédés après un arrêt cardiaque contrôlé a été établi en 1992 au Centre Médical de Pittsburgh. Aux États-Unis, les donneurs de cette catégorie constituent l'essentiel des prélèvements réalisés chez les donneurs en arrêt cardiaque (Steinbrook, 2007). Ces prélèvements ont donné lieu, en 2006, à 645 interventions.

Actuellement, aux États-Unis dans le programme de la « New England Organ Bank », les patients concernés sont, pour un tiers, des patients traumatisés crâniens par accident sur la voie publique (AVP), pour un autre tiers, des patients ayant eu un accident vasculaire cérébral (AVG) et enfin des patients anoxiques. A ces patients inconscients et gravement cérébrolésés doivent être ajoutés ceux pour lesquels l'arrêt des thérapeutiques procède d'une volonté 
exprimée par le malade. Il s'agit alors de patients conscients demandant l'arrêt de la ventilation mécanique dont ils dépendent entièrement, tels les patients tétraplégiques dont la lésion est très haute (G1 et $\mathrm{C} 2$ ), les patients ayant une sclérose latérale amyotrophique (SLA) et ventilés au long cours, les patients souffrant d'une insuffisance respiratoire chronique ventilée. Ces patients entrent dans la catégorie des donneurs potentiels DDAC III aux États-Unis et en Belgique.

\subsection{La définition de la mort a une incidence sur le type de prélèvement autorisé}

Le prélèvement d'organes, s'agissant des patients DDAC III, témoigne d'une divergence sur les normes fondamentales qui encadrent les pratiques. La variabilité des normes, pourtant établies comme des normes de référence, s’illustre en premier lieu dans les critères auxquels il est fait référence pour déclarer la mort d'un patient: ceux-ci ne font pas l'objet d'une unanimité dans le monde occidental. Concernant l'état de mort encéphalique, il est défini dans les pays anglo-saxons en référence à la destruction du tronc cérébral (lower brain-death) alors qu'en France et dans d'autres pays comme l'Espagne, on se fonde sur la destruction totale du cerveau (whole brain-death). Dans le cas des patients DDAC, on observe une variabilité internationale du délai légal entre l'asystolie et la signature du certificat de décès. Ce délai est de $2 \mathrm{mn}$ à Pittsburgh, $5 \mathrm{mn}$ au Canada et en France, $10 \mathrm{mn}$ dans d'autres États des États-Unis et en Grande-Bretagne, 20 mn en Suède. Là où le prélèvement d'organes sur patients DDAC III est autorisé, les critères auxquels il est fait référence pour déclarer la mort du patient ont aussi fait l'objet de discussions. Ainsi aux États-Unis, on s'est demandé si l'arrêt de la circulation après $5 \mathrm{mn}$ était suffisant pour garantir la condition d'irréversibilité du Uniform Determination of Death Act (UDDA) de 1981 qui définit la mort soit comme la cessation irréversible des fonctions circulatoires et respiratoires, soit comme la cessation irréversible de toutes les fonctions du cerveau. Ce délai a été fixé 10 mn dans certains États des États-Unis et en Grande-Bretagne.

La notion d'« irréversibilité » s'entend, dans la pratique, en un double sens. On parle d'irréversibilité «forte » face à un arrêt cardiorespiratoire et à un état de mort encéphalique, persistant au-delà des efforts déployés pour restaurer la circulation corporelle, la respiration et les fonctions cérébrales. Dans ce cas, on constate l'irréversibilité alors même que toutes les mesures ont été prises pour tenter la réanimation. En revanche, on parle d'irréversibilité « faible », lorsque celle-ci est définie par l'intention des médecins de ne pas procéder à une réanimation. On considère alors que la réanimation ne doit pas être entreprise plutôt qu'elle ne 
le sera pas (voir Bernat 2008, Curfman et al. 2008, Veatch 2008, Truog et Miller 2008)4. Cette interprétation «faible » de l'irréversibilité prévaut aujourd'hui. Nous verrons alors, que pour des raisons de préservation des organes en vue d'un prélèvement, la distinction entre « patient mort » et « patient sur le point de mourir » tend à s'estomper.

Ces éléments montrent que la description d'un état, ici anthropologiquement fondamental comme la mort ou l'irréversibilité d'un processus morbide, et le choix des critères en fonction desquels cette description est faite ont des conséquences sociales et éthiques décisives. L'adoption de normes spécifiques mais contingentes (i.e. la référence à la destruction du tronc cérébral plutôt qu'à la destruction totale du cerveau, dans tel contexte national) ainsi que les termes dans lesquels sont décrits certains «états du monde» autorisent des pratiques moralement controversées ${ }^{5}$. Elles sont également permises par la qualification - à travers la notion d'irréversibilité par exemple - d'états partiels autour desquels le consensus n'est pas acquis.

L'indétermination des normes induit et autorise des divergences entre les pratiques - ici reconnues comme acceptables, là stigmatisées comme intolérables, ou bien aujourd'hui admises et demain abandonnées, pour des raisons morales - ainsi que des dilemmes moraux irréductibles. Ces divergences suscitent des phénomènes de concurrence entre les normes, dans un contexte élargi de surenchère entre les pays sur le nombre de greffes réalisées chaque année $^{6}$. Or l'adoption d'un point de vue moral récuse qu'il faille nécessairement et

\footnotetext{
${ }^{4}$ Pour une discussion des notions d'irréversibilité et de cessation permanente, voir Bernat, 2006a, p. 124-126. Bernat suggère d'envisager l'irréversibilité en référence à la "permanence », la première étant alors entendue comme la cessation permanente des fonctions respiratoires et circulaires, durant une période d'observation appropriée. La mort des donneurs DCD advient lorsque la respiration et la circulation ont cessé et que les fonctions cardio-pulmonaires ne reprennent pas spontanément. L'«irréversibilité » devient synonyme d'un arrêt «permanent» de la respiration et de la circulation. En effet, lorsque l'on a établi que la perte des fonctions respiratoires et circulaires est permanente, elle devient rapidement et inévitablement irréversible au bout des quelques minutes après lesquelles le cerveau est détruit par manque d'oxygène et de circulation sanguine. Dans ce contexte, la «permanence » représente une étape préliminaire d'un processus inévitable qui conduit inévitablement et sans doute possible (i.e. certainement) à l'irréversibilité. Néanmoins dans le contexte du DCD, les fonctions respiratoires et circulaires du patient peuvent avoir cessé de façon permanente sans qu'elles aient cessé de façon irréversible (Bernat, 2006b, p. 282).

${ }^{5}$ Ainsi la détermination d'un état comme la mort encéphalique a permis d'ouvrir un nouveau champ pour le prélèvement d'organes. Les pratiques se situent de part et d'autres d'une ligne évanescente de partage entre un concept descriptif de la mort et un critère évaluatif de la mort. La mort cérébrale est introduite en droit français par la circulaire Jeanneney du 24 avril 1968 qui légalise le prélèvement d'organes sur ce type de sujet. Or cette définition, dont certains récusent encore qu'elle traduise la réalité de la mort, a été établie pour légaliser les prélèvements d'organes et pour autoriser l'arrêt du respirateur de ces patients, qui n'existaient pas avant le développement de la ventilation artificielle. Telle forme de description d'état du monde autorise telle pratique. Il y a, dans la déclaration du décès, une dimension performative (voir Hennette-Vauchez et Nowenstein, 2009, p. 42).

6 Voir notamment sur la surenchère espagnole sur les prélèvements d'organes et les stratégies visant l'optimisation de la production des organes à greffer (Steiner, 2006). Cette perspective signifie également que l'évolution des normes françaises dans le domaine pourrait avoir lieu du fait d'une adaptation des pratiques dans d'autres pays occidentaux.
} 
systématiquement réagir à des situations nouvelles soit par des réaménagements normatifs, c'est-à-dire par l'adoption de nouvelles normes ou de nouvelles interprétations des normes acceptées, soit par des réaménagements dans les valeurs de référence, encadrant l'action ou à l'œuvre dans les organisations (Guibet Lafaye et Picavet, 2010). Tel est le cas par exemple, concernant le prélèvement d'organes, pour la règle du «donneur mort » ainsi que nous le verrons ultérieurement en détail.

Les critères en référence auxquels la mort est définie ainsi que les attitudes nationalement assumées à l'égard de l'euthanasie - en somme, les phénomènes d'autorisation antécédents ont des incidences sur les limites que l'on s’impose dans les pratiques de prélèvement d'organes7. D’une part, les pays qui définissent l'état de mort encéphalique par la mort du tronc cérébral acceptent plus volontiers le prélèvement d'organes sur patients DDAC III. D'autre part, dans des pays où l'euthanasie est légalisée, on constate que le prélèvement d'organes s'oriente, de façon privilégiée, vers des patients DDAC III puisqu'ils représentent 82\% des prélèvements à cœur arrêtés en Angleterre, 84\% en Belgique, et 93\% aux Pays-Bas. La question de l'extension du prélèvement d'organes aux patients DDAG III ne peut être envisagée exclusivement comme une question théorique - d'éthique pure - ou de droit car elle impose que la réflexion morale prenne en compte les effets empiriques induits par une telle autorisation.

\section{La fin justifie-t-elle les moyens ?}

\subsection{Bénéfices escomptés de l'autorisation d'une pratique aujourd'hui controversée}

Quel serait le nombre de greffons susceptibles d'être recueillis, si le prélèvement d'organes était autorisé sur les patients de la classe III de Maastricht? Combien de sujets potentiels seraient-ils concernés en France, si l'on tient compte de la faible représentation des patients cérébrolésés - sans défaillance multiviscérale ni pathologie maligne - parmi les patients pour lesquels un arrêt des thérapeutiques actives est décidé aujourd'hui en réanimation? Quels bénéfices faudrait-il obtenir, pour juger acceptable une évolution des pratiques médicales, tenue par certains pour transgressive? Se justifie-t-elle, par exemple, pour 600 greffons par an, nombre de greffons que l'on obtiendrait en réduisant le taux de refus de prélèvement des patients donneurs en état de mort encéphalique (DDME) de 30 à 20\% ?

${ }^{7}$ La France a, pour sa part, se tient en marge des choix en faveur de l'euthanasie active, avec la loi $\mathrm{n}^{\circ}$ 2005-370 du 22 avril 2005, relative aux droits des malades et à la fin de vie ouvrant en effet une autre voie. 
Le nombre de greffons escomptés ne pourra être évalué qu'une fois qu'auront été déterminés avec précision les types de patients éligibles pour cette procédure. Dans une hypothèse limitative, par exemple, c'est-à-dire si le prélèvement sur patients DDAC III n'est autorisé que pour les patients dans le coma par anoxie cérébrale, après arrêt cardio-

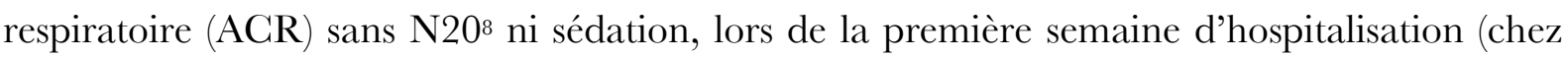
qui la certitude d'un mauvais pronostic est de l'ordre de 100\% (voir Fisher et al., 2004, Fisher et al., 2006), ils ne seront que quelques centaines. Dans une hypothèse extensive, ils pourraient être beaucoup plus nombreux si l'on incluait, dans le prélèvement, les patients cérébrolésés ayant un mauvais pronostic neurologique (état végétatif ou pauci-relationnel). Cette inclusion large soulèverait cependant des dilemmes moraux que nous allons explorer. Dans ce cas en effet, la survenue rapide de l'arrêt cardiaque devra être favorisée, d'une façon ou d'une autre, car le temps d'ischémie chaude ${ }^{9}$ risque d'être trop long pour permettre un prélèvement d'organes ${ }^{10}$. L'autre solution serait d'abandonner la «dead donor rule », voire d'autoriser le prélèvement cardiaque ou le prélèvement multi-organes sans arrêt cardiaque préalable.

\subsection{Provoquer la mort pour préserver les organes?}

La possibilité du prélèvement d'organes sur patients DDAG III et la préservation des organes en vue d'une greffe ultérieure imposent des conditions et des gestes spécifiques, induisant des dilemmes moraux, qui ne se rencontrent pas dans le prélèvement d'organes sur patients DDAC I et II. Pour les patients, pour lesquels un retrait des thérapeutiques actives est décidé, s'ouvre une phase où la mort par arrêt cardiaque du malade est attendue. Elle est associée à une lente dégradation de la fonction cardio-circulatoire, à l'origine de conséquences organiques systémiques afférentes qui provoquent une détérioration progressive des organes potentiellement prélevables. Le temps moyen après lequel survient la mort des patients en réanimation, candidats pour le prélèvement après arrêt cardiaque est de $5 \mathrm{~h}$ (Inter-quartile range 1.4-11.5h), après le retrait des traitements de support vital (Revelly et al., 2006). Cette durée dépasse les $90 \mathrm{mn}$ voire les $60 \mathrm{mn}$ d’ischémie chaude, requises pour le prélèvement.

\footnotetext{
8 Il s'agit d'une réponse électrophysiologique à une stimulation auditive. Son absence chez un patient ne présentant pas de facteurs confondants (surdité, sédation, hypothermie...) est de très mauvais pronostic.

${ }^{9}$ C'est-à-dire le temps qui sépare le débranchement du respirateur de l'arrêt du cœur, temps durant lequel la perfusion des reins et du foie se fait mal, avec une réduction du débit et de l'oxygénation artérielle.

${ }_{10}$ Ceci ne sera cependant envisageable que lorsque des techniques parfaitement fiables et validées au plan international de pronostication du devenir neurologique, utilisables rapidement dans le cours évolutif de la maladie, auront été mises au point.
} 
L'élément crucial réside donc ici dans le laps de temps au-delà duquel les organes du donneur se seront si dégradés qu'ils ne seront plus transplantables.

La capacité de prévoir le temps avant la mort par arrêt cardiaque du patient, dont on a limité les thérapeutiques et après que les traitements de support vital ont été retirés, est donc décisive. Or on constate que, dans $33 \%$ à $50 \%$ des cas aux États-Unis, le patient ne décède pas après le retrait des traitements de support vital, dans le temps requis pour préserver la qualité des organes en vue d'un prélèvement ${ }^{11}$. Dès lors, se pose la question de savoir, dans une logique de maximisation du bénéfice social, s’il est légitime ou pas de raccourcir cette période d'ischémie chaude afin de préserver la fonctionnalité des organes ${ }^{12}$. En somme, la fin justifie-t-elle les moyens?

Dans la mesure où le patient va mourir, est-il légitime d'accélérer sa mort en vue de préserver ses organes? Peut-on s'autoriser des actes qui abrègeront l'agonie pour sauver ses organes et les rendre disponibles à la greffe ? Ne transgresse-t-on pas une règle socialement fondamentale selon laquelle aucune vie humaine ne doit être sacrifiée au profit d'une autre? Le questionnement éthique s'inscrit ici dans une problématique qui le porte au-delà de la distinction, qui a souvent servi de référence dans les débats sur la législation de l'euthanasie, entre «s'abstenir », d'une part, et « provoquer » ou « agir intentionnellement », d'autre part. Peut-on justifier, d'un point de vue moral, une logique du type «puisqu'il va mourir, alors... » que l'on trouve déjà mise en œuvre dans certains pays occidentaux (Vincent, 2010)? A quelles objections un raisonnement strictement utilitariste ou de pure maximisation du bénéfice social, qui autoriserait ces actes, se voit-il confronté13 ?

Certaines équipes, dans des pays où ce type de prélèvement est autorisé, engagent des procédés qui accélèrent le processus induisant l'arrêt cardiaque. Aux États-Unis, des prélèvements cardiaques ont été réalisés sur trois nouveaux-nés en anoxie cérébrale (Boucek et al., 2008), extubés sous sédation «standardisée »"15 et décédés d'arrêt cardiaque. En Belgique, l'extubation des patients est parfois faite sous curare ${ }^{16}$ (voir Ysebaert et al., 2009). Cette accélération du processus morbide - qui peut passer par le recours à divers types de

\footnotetext{
${ }^{11}$ Données non publiées de la New England Donor Databank.

12 Plus techniquement, se pose également la question de savoir combien de temps, une fois que le cœur de la personne s'est arrêté, il faut attendre avant d'être sûr que cet arrêt soit considéré comme irréversible et que le décès puisse être légalement certifié.

${ }^{13}$ Rappelons que le droit français autorise des exceptions au principe d'indisponibilité du corps humain et de ses produits (Code Civil, art. 16 sq.) pour certaines cessions dont les conditions sont imposées par la loi et lorsqu'une finalité thérapeutique est avérée.

${ }^{15}$ Adaptée au poids et non pas à l'état du nouveau-né.

${ }^{16}$ Le curare supprime toute fonction motrice musculaire et paralyse les muscles inspiratoires. Sous curares mais sans ventilation artificielle, la mort survient en 5 à 10 mn. Le cœur s'arrête par manque d'oxygène.
} 
techniques (sédation, hypotenseurs - comme on le fait aux États-Unis -, voire curares) permet de raccourcir le temps d'ischémie chaude et de préserver les organes du donneur en vue de leur prélèvement.

Alors que dans le cas des patients DDAC I et II, rien d'intentionnel n'intervient dans la causation de l'arrêt cardiaque, tel n'est pas le cas pour les patients DDAC III. On impose alors, en lieu et place d'une évolution, de type spontané (ou naturel) vers la mort, une évolution intentionnelle induite. Dans le cas du prélèvement d'organes sur patients DDAC III, l'exigence de la préservation des organes ne permet pas que la dégradation spontanée des fonctions organiques conduise, par elle seule, à la mort. La fin de vie (i.e. les dernières heures ou jours) du patient ne se trouve alors plus envisagée de façon immanente et du point de vue de la totalité signifiante qu'elle représente en tant que telle (Aristote, Ethique à Nicomaque) et pour ses proches. Une intentionnalité de second rang, i.e. ne concernant pas directement l'individu sur lequel les actes sont réalisés, s'introduit.

\subsection{Limites intrinsèques de l'argument utilitariste}

On pourrait considérer que l'accélération du processus morbide pour préserver les organes en vue du prélèvement se justifie par l'utilité sociale que représente la greffe. La logique est alors celle du rapport coût/bénéfice. L'argumentation morale se voit mise en concurrence avec des arguments d'une autre nature. A l'argumentaire de la « pente glissante » font face les arguments utilitaristes et d'efficacité dont on présuppose souvent l'incontournabilité et l'irrévocabilité. Pourtant la maximisation des bénéfices - sociaux en l'occurrence - n'est pas une stratégie qui doive prévaloir dans tout type de situation, pas plus que l'argument utilitariste n'a une priorité systématique sur tout autre type d'argument, notamment moral. La référence à l'efficacité ne suffit pas à justifier des pratiques moralement transgressives. Dès lors que l'on accepte de se placer dans un référentiel moral, les arguments d'efficacité ne peuvent plus réellement être mis en balance avec des arguments éthiques (Tugendhat, 1998, p. 9-10).

Comment justifier, d'un point de vue éthique, que l'on puisse abréger une vie pour prolonger la durée d'une autre vie et sa qualité ? Peut-on hiérarchiser les vies, en considérant que l'une étant proche de son terme, sa prise en compte doit être relativisée, au motif notamment que «puisqu'elle atteint son terme, alors...»? Une position favorable à une intervention active sur la fin de vie du donneur témoigne d'une attitude spécifique à l'égard de la mort, de la fin de vie et de la qualité de vie, et engage une définition sociale de la personne. 
Elle fait, pour une part, prévaloir des considérations pragmatiques et utilitaristes ${ }^{20}$ sur des considérations morales. Le statut donné au corps, y compris lorsqu'il s'agit du corps d'un mourrant, exprime quelque chose du statut conféré au sujet et à la personne (voir Lebreton, 2008, p. 17). On pourrait considérer que la préférence pour le «sauvetage des organes» contrevient, dans certains cas que nous allons évoquer, à des normes fondamentales telles que le principe de non malfaisance, de respect de l'intégrité physique et de l'autonomie du patient ${ }^{21}$.

Enfin, l'accélération du processus morbide, lorsqu'elle aura lieu, sera le fait de soignants. Elle impose des coûts psychiques dont la réalité ne peut être oblitérée et doit être prise en compte, y compris lorsque l'on se place dans un calcul strict des coûts/bénéfices associé au prélèvement d'organes sur patients DDAC III. L'extension du champ d'applicabilité des normes - i.e. l'inclusion des patients DDAC III dans l'autorisation du prélèvement d'organes ne constitue pas simplement une question de droit ou d'élargissement de la législation mais comportera très probablement des effets dérivés dommageables, pour ceux qui seront en charge de la réalisation des actes concernés. Ces acteurs pourraient en effet se trouver lésés dans le respect de leurs convictions morales personnelles.

Du simple point de vue du rapport coûts/bénéfices, la souffrance des soignants, dans ces situations, ne doit pas être sous-estimée (voir Verheijde et al., 2007, p. 3). Elle est déjà documentée dans des situations de fin de vie, où l'évolution du patient suscite moins de discussions éthiques. Certaines études attestent, parmi les soignants, le sentiment que pour les patients DDAG III, l'état de mort n'est pas atteint (D'Alessandro et al., 2008, p. 1079 ; Lebreton, 2008, p. 289-291). Les actes qui accélèrent l'évolution vers la mort du patient comportent en effet des coûts indéniables pour le personnel soignant (Siminoff et al., 2004), y compris en termes de préservation et de respect de leurs convictions morales personnelles. $\mathrm{Si}$ l'on accepte d'outrepasser, comme nous le préciserons dans ce qui suit, la règle du donneur mort, en somme si les organes peuvent être prélevés sur des patients dont le pronostic est une mauvaise évolution neurologique et pour lesquels le prélèvement aura des incidences sur l'advenue de la mort, il faudra déterminer comment seront alors réalisés les actes requis.

\footnotetext{
${ }^{20}$ Rappelons toutefois que le fait de prélever un organe ne garantit pas le succès de la greffe. En somme, le prélèvement d'un rein ne signifie pas, symétriquement, qu'un patient pourra échapper à la dialyse.

${ }_{21}$ Nous envisagerons ultérieurement le cas du patient qui pourrait consentir à un arrêt des thérapeutiques, associé à un don d'organes.
} 


\subsection{L'organisation des pratiques et les risques de dérive}

L'inclusion de patients DDAC III dans le prélèvement d'organes peut donner lieu - plus que pour les patients DDAG I et II (voir Guibet Lafaye et Puybasset, 2010) - à des conflits entre professionnels de santé ${ }^{4}$. Non seulement la mort survient dans le cadre d'un processus de «withdrawing » (i.e. de retrait actif des thérapeutiques) mais aucun des patients cérébrolésés concernés n'a comme alternative à l'extubation la mort. Dans la très grande majorité des cas, l'alternative est un état pauci-relationnel ou plus rarement un état végétatif. Le souci d'un recueil optimisé des greffons suppose un recensement des patients donneurs potentiels qui suscite des dilemmes éthiques évidents. Pourtant certaines institutions hospitalières ont autorisé que des coordinateurs d'institutions de prélèvements procèdent à des recensements, en réanimation, avant tout recueil du consentement (pour le prélèvement) et sans que les familles ne soient informées de la démarche (Salim et al., 2007). L’influence des centres de transplantation est patente dans le système américain où existent déjà des « Organ Procurement Organizations ». On observe également, dans certains cas, des pressions des centres de soins et des centres associés aux industries liées aux soins de santé (Ranjan et al., 2006). Les intérêts liés au développement de programmes de transplantation peuvent également éloigner de l'éthique des soins, de l'éthique palliative et de la déontologie des services de réanimation. L'ensemble de ces conflits d'intérêts peut être source de pressions sur les soignants et les équipes.

L'évolution des normes encadrant les pratiques, sous l'effet notamment de groupes de pression comme les associations de patients dialysés ou des programmes de transplantation, comporte des coûts externalisés sur les individus, directement impliqués par le processus de prélèvement. Elle ne peut être envisagée exclusivement en termes de bénéfices associés à la greffe pour les transplanteurs, les receveurs et leurs familles. Une approche qui serait exclusivement conséquentialiste appelle cette prise en compte large des coûts induits par l'extension du champ des candidats au prélèvement. Alors que le rapport coûts/bénéfices est positif, dans le cas du prélèvement sur patients en état de mort encéphalique et pour les patients DDAG I et II, il se pourrait bien que le jeu soit à somme nulle voire à somme négative, pour les patients DDAC III (Guibet Lafaye et Puybasset, 2010).

De même, si l'on devait satisfaire exclusivement le seul critère d'efficacité, sans autre considération morale, le prélèvement sur personnes en état de mort encéphalique s'avèrerait

\footnotetext{
${ }^{24}$ Sur les dérives potentielles du prélèvement d'organes sur patients DDAC III, voir Bayley (2009). Carol Bayley, dans une étude du Catholic Healthcare West montre qu'en Californie, 35000 personnes se trouvent en état végétatif et que deux tiers des neurologues approuveraient un prélèvement sur ces patients.
} 
le plus «efficace ». D’une part, il est largement accepté par la société française et surtout, d'autre part, les procédures de prélèvements actuelles en France sont sous optimales (Riou 2010). En d'autres termes, la référence à l'efficacité sur laquelle pourraient s'appuyer les partisans du prélèvement sur patients DDAG III n'est pas univoque. Ainsi pourrait être privilégiée, dans le cadre du dispositif actuel, l'optimisation du système de prélèvement - avec un maillage territorial homogène et resserré, associé à un investissement financier importantplutôt que l'élargissement du cercle des donneurs potentiels. On sait en particulier que plusieurs milliers de patients pourraient entrer dans les procédures DDAC I et II, qui sont parfaitement adaptées au système de santé français, en particulier du fait des réseaux d'urgences et de SAMU. Cette optimisation du système existant éviterait enfin la transgression de principes moraux fondamentaux.

\section{Traitement des corps et réévaluation du respect de la fin de vie}

\subsection{L'instrumentalisation du corps du donneur et de sa fin de vie, justifiée par les bénéfices escomptés de la greffe}

S'agissant des patients DDAC III, un conflit d'intérêt majeur entre donneur et receveur est suscité par la possibilité qu'une abstention («withholding») ou un retrait thérapeutique («withdrawing ») soit décidé chez un patient, dont on ne pourrait affirmer définitivement, avec un très haut degré de certitude le mauvais pronostic ${ }^{25}$, et chez lequel ce retrait serait accompli uniquement pour obtenir des organes. Ces conflits se donnent à saisir dans les interrogations et dilemmes moraux suivants : tout ce qui devait être fait pour le patient a-t-il été fait? On peut parer à ce premier doute, lorsque les proches peuvent être les témoins de ce que tout ce qui pouvait être fait pour le patient l'a été. Reste à savoir si les intérêts vitaux du donneur potentiel ont été pris en compte sans que ne prévale le souci d'un potentiel prélèvement. Ce donneur a-t-il bien été considéré comme une fin dans le processus thérapeutique ? Certains mettent déjà en évidence une tendance internationale à faire prévaloir les intérêts du receveur sur ceux du donneur et son consentement, y compris pour les patients n'entrant pas dans la catégorie DDAC III (Spital, 2003). L'étanchéité entre les services et les spécialités (les services de réanimation ou de soins palliatifs, où sont décidés les arrêts des thérapeutiques, d’une part,

\footnotetext{
${ }^{25}$ La décision du prélèvement sur un patient, pour lequel il n’y a plus d'espoir, doit advenir à un moment où le diagnostic peut être fait avec certitude. Or une décision d'extubation ne peut être prise rapidement que dans quelques cas spécifiques comme pour les patients anoxiques. Avant le troisième jour de prise en charge, aucun pronostic des lésions cérébrales ne peut être établi avec certitude.
} 
et les services où sont réalisées les greffes, d'autre part) est une condition éthique du respect du donneur mais dont on sait qu'elle est loin d'être toujours garantie dans les faits.

La question de la finalité en vue de laquelle les soins sont réalisés sur le donneur potentiel est également lourde de conflits moraux: les actes pratiqués - i.e. ceux esquissés précédemment - le sont-ils en vue d'augmenter les opportunités de dons ? Un dilemme moral peut en effet se poser, lorsque les équipes de réanimation favorisent une obstination thérapeutique (plutôt qu'une limitation des thérapeutiques, alors même qu'elles sont persuadées de la futilité de la réanimation), dans le but de mieux contrôler la temporalité de la mort et de prolonger le temps durant lequel envisager, avec les proches, le don, tout en préservant les organes. D'ores et déjà des pratiques institutionnelles, identifiables liées à certains facteurs - autres que les caractéristiques du patient, sa maladie, ses préférences ${ }^{26}-$ tendent à commander l'utilisation de soins de supports vitaux plutôt que le recours à des soins palliatifs en fin de vie (Zingmond et al., 2005). On initie alors un processus d'obstination thérapeutique stratégique, visant à maximiser les potentialités du don. De même, il est techniquement possible de favoriser la mort encéphalique de ce type de patients, aux dépens de la mort cardiaque, et par conséquent de susciter - plutôt que pas - les conditions légales requises pour le prélèvement d'organes (voir Lara, 2000). En a-t-on le droit ? Conformément à quels principes? Il est certain que s'opère ici a minima une inversion de finalité ainsi qu'une inversion dans le regard porté sur le patient et la façon dont il est envisagé : de patient, il devient donneur (potentiel) et sa fin de vie est prise en charge en vue d'un autre que lui-même et non plus conformément à une perspective immanente à son propre devenir. Ce changement de perspective ne correspond-il pas à une définition stricte de l'instrumentalisation?

Comme nous l'avons souligné en introduction, l'approche qui prévaut et le discours dominant concernant le don d'organes se formulent aujourd'hui, de façon récurrente, en termes de « pénurie » qui introduit, sans plus de médiation, la réflexion en la matière dans une logique de marché. Les organes sont assimilés à des biens circulant dans un marchée ${ }^{28}$ et passant de donneur à receveur par le biais de circuits institutionnalisés. Cette orientation qui prévaut, dans le milieu médical et pour certaines associations de la société civile, nourrit une représentation parcellisée du corps humain, envisagé comme une somme d'organes dissociables (voir Lebreton, 2008, p. 325-326) et d'autant plus segmentés que la mort du

\footnotetext{
${ }^{26}$ Mais relatifs à des caractéristiques institutionnelles, aux normes hospitalières ou propres à chaque service, à la disponibilité du personnel soignant, à des intérêts particuliers, etc.

${ }^{28}$ L'évocation d'un «marché » est d'autant plus justifiée que des prestations financières accompagnent souvent, dans des pays comme l'Espagne, le prélèvement d'organes.
} 
patient se fait proche. L'individu n'est plus - ou plus seulement - perçu comme une personne en train de mourir mais comme une ressource d'organes à préserver et pouvant être prélevés. S'amorce une évolution du statut anthropologique du mourant et du corps proche de la mort.

\subsection{Respect de l'éthique palliative vs. maximisation de la qualité de vie des receveurs}

Le conflit d'intérêts entre donneurs et bénéficiaires du prélèvement - qui se formule également comme une opposition entre logique de marchandisation et éthique palliative - est accru, lorsque le donneur n'est pas un patient cérébrolésé mais se trouve en unité de soins intensifs. Ce conflit s'exacerbe, lorsque le patient décède non plus d'un arrêt des thérapeutiques actives mais des suites d'une euthanasie active, réalisée de la main d'un soignant comme on en trouve des exemples en Belgique ou en Californie, à travers des cas de suicides assistés de patients porteurs de pathologies neurologiques chroniques, telles que la sclérose latérale amyotrophique (SLA), la sclérose en plaques ou de dons provenant de patients tétraplégiques $\mathrm{C} 1-\mathrm{C} 2$, réclamant un arrêt de traitement, par exemple. On ne peut sous-estimer, dans ces processus de fin de vie, la force d'influence du médecin sur son patient (Pelluchon, 2009). Le risque existe donc que la mise en œuvre de la pratique du DDAC III, dans les hôpitaux, creuse un fossé entre la garantie et la délivrance de soins compassionnels aux patients, en fin de vie et en phase terminale de leur maladie, et le bénéfice qui peut être tiré du prélèvement d'organes sur ces patients.

Le fait que le patient ne soit plus appréhendé seulement comme un patient en train de mourir mais comme un donneur potentiel suscite des actes, pratiqués sur son corps, dont il n'est plus le bénéficiaire exclusif. Ainsi le retrait de l'assistance vitale peut être reporté ou retardé soit jusqu'à ce que le critère de la mort encéphalique soit vérifié (afin, par exemple, de prélever le cœur du patient en plus d'autres organes) soit jusqu'à ce que les fonctions cardiorespiratoires se soient dégradées, de telle sorte que la mort suive rapidement le retrait de l'assistance vitale. Or ces pratiques ont une incidence négative sur la temporalité et la qualité des soins palliatifs de fin de vie. Le patient en serait préservé, s'il n'était pas appréhendé comme un donneur potentiel. Deux logiques se trouvent mises en concurrence dont l'une fait du patient une occasion - ou un point d'appui - d'un processus dont la finalité lui est extérieure.

De même, on retrouve, avec les patients DDAC III, l'un des dilemmes auxquels sont confrontées les équipes, pour les patients DDAC I et II. Dans les deux cas, existe un décalage entre la réalisation de gestes ou l'administration de substances, préservant la possibilité du 
prélèvement d'organes (mise en place des sondes de Gillot ou d'une circulation régionale normothermique ${ }^{29}$ ), sans que la position du donneur potentiel ne soit connue ni que ses proches aient été consultés. Deux types de gestes sont à distinguer: d'une part, l'administration de traitements avant la déclaration du décès et, d'autre part, l'administration de traitements avant d'avoir vu les familles. Or la réalisation de gestes ou l'administration de substances au patient DDAC III dont il n'est pas le bénéficiaire sont nécessaires, si l'on veut que ses organes puissent être prélevés ${ }^{31}$. Le risque que la nécessité de fournir des organes utilisables nuise à la qualité et au type de soins, donnés en fin de vie, est enfin supérieur dans le prélèvement sur patients DDAC III, pour les raisons que nous avons évoquées et qui ne se rencontrent pas s'agissant des patients DDAG I et II. Se trouvent placés en concurrence les intérêts du donneur et ceux du receveur potentiel, les seconds se voyant accorder une priorité sur ceux du premier, alors même que ce dernier est le support des actes réalisés. Ce conflit des finalités, qui tend à faire prévaloir une amélioration potentielle de la qualité de vie des receveurs sur le respect de la fin de vie des donneurs, trouve une autre issue dans des pays qui, comme le Japon, ont adopté des législations respectueuses de la diversité des interprétations de la mort ${ }^{32}$.

La possibilité du prélèvement a très largement modifié le traitement des patients, des corps et leurs représentations. La perspective du don d'organes altère, incontestablement, le contexte de la mort de la personne, ce qui a également des incidences sur ce que vivent, dans ces moments difficiles, ses proches. Se pose par exemple la question du déplacement du patient vers la salle d'opération : l'emmène-t-on avant ou au moment même de l'annonce de la mort pour initier, sans délai, le processus de prélèvement d’organes? Plus généralement, assume-t-on de ne pas laisser la famille avec le patient qui vient de mourir ou de ne pas la laisser accompagner le malade jusqu'à la mort parce que ses organes doivent être prélevés et qu'il doit donc être conduit en salle d'opération? Respecte-t-on ici encore le principe de non

\footnotetext{
${ }^{29}$ Cette circulation extracorporelle vise à perfuser les reins et le foie durant la discussion avec les proches.

${ }^{31}$ Administration d'héparine par exemple.

${ }^{32}$ Le Japon est le seul pays qui autorise les individus à choisir leur définition de la mort, cardiaque ou cérébrale, en matière de prélèvement d'organes, en accord avec les membres de leur famille. Elle est inscrite au dos de leur carte de donneur («Organ Donation Decision Card»). Jusqu’à la fin des années 90, la mort encéphalique n’était pas légalement reconnue dans ce pays. Cette disposition légale ne signifie pas que les individus élisent une définition arbitraire de la mort puisque leur choix s'opère entre des définitions établies de la mort (mort cérébrale, mort cardiaque auquel s'ajoute le refus du don). La tradition culturelle japonaise, le rapport aux corps et aux défunts ont pour effet que la majorité des greffes de reins, contrairement à ce que l'on connaît dans le monde occidental, est réalisée à partir de donneurs vivants. La technologie des prothèses s'y est aussi considérablement développée.
} 
malfaisance, fondateur de la pratique médicale ${ }^{33}$ ? L'autorisation d'une telle pratique n'offre aujourd'hui aucune garantie quant au respect des principes de non malfaisance, de l'intégrité physique du patient ou de l'autonomie du malade. Elle illustre un cas bien connu de sacrifice de l'utilité individuelle au bénéfice de l'utilité globale espérée de l'ensemble social (puisqu'un patient est susceptible de fournir plusieurs greffons) (voir Rawls, 1971, p. 51-52). Ces pratiques, éthiquement problématiques, doivent a minima pouvoir valider un critère de publicité : peut-on justifier auprès de tiers les actes pratiqués, en vue d'un prélèvement d'organes, sur les patients DDAC III ? Elles doivent satisfaire l'exigence de pouvoir être clairement expliquées et justifiées devant un soignant, un proche, un juge comme l'exige par exemple la loi du 22 avril 2005.

\section{Consentement et autonomie}

\subsection{Valeur et légitimation des pratiques par la référence au consentement}

Si le consentement était la clef de l'autorisation de ce type de prélèvement, se poserait inévitablement la question de la fréquence à laquelle le consentement devrait être renouvelé, de la part de ces patients conscients ainsi que celle de sa nature. Quel type de consentement doit-on retenir? Faut-il retenir l'interprétation souple du consentement présumé, où le donneur n'a déclaré aucune opposition au prélèvement mais qui n'exclut pas que l'avis des proches soit considéré35 ? Que fera-t-on pour les personnes n'ayant pas la capacité de décider et pour les personnes dont on ignore l'identité ? La demande de renouvellement de consentement n'a-t-elle pas des effets incitatifs propres - effaçant, de la sorte, la neutralité de

\footnotetext{
${ }^{33}$ Se pose ici la question de la concurrence entre les bénéficiaires (donneur et receveur) d'actes pratiqués sur le corps du donneur et de la comparabilité de leurs utilités. On cherche à balancer une possible malfaisance à l'égard du donneur avec une possible bienfaisance pour le receveur mais on peut douter - comme suggère la tradition économique - qu'existe une réelle commensurabilité entre leurs utilités personnelles. On met également en concurrence et tente de comparer les coûts supportés par les proches du patient qui va mourir avec ceux assumés par les proches du patient en attente de greffe. S'agissant des familles confrontées à l'état de mort encéphalique d'un proche ou d'un familier, les conséquences du prélèvement ne sont pas clairement identifiées comme négatives car le don peut jouer un rôle favorable dans le processus de deuil. Les effets négatifs sont souvent considérés comme étant compensés par le bénéfice social du don d'organes. Bien que ces coûts n'aient pas été évalués pour les proches de patients DDAC I et II, ils semblent limités. L'absence d'évaluation prévaut néanmoins pour les familles de patients DDAC III.

${ }^{35}$ En France, prévaut la règle du consentement implicite qui constitue une référence pour le prélèvement d'organes sur des patients en état de mort encéphalique ou DDAC I et II. Dans les faits, ce sont le plus souvent les proches qui prennent la décision.
} 
la procédure ? Plus fondamentalement, l'obtention et la référence au consentement suffisentelles à justifier la pratique envisagée et son acceptabilité morale $^{37}$ ?

Dans le cas des patients DDAC III, en particulier conscients, plusieurs questions se posent : comment garantir l'autonomie du patient dans ces conditions? Dans quelle mesure le consentement du patient conscient, qui demande l'arrêt de son support ventilatoire, est-il vraiment éclairé ou informé ? Ne transgresse-t-on pas les limites légales de l'autonomie, dans la mesure où personne ne pourrait consentir à sa propre mort (Verheijde et al., 2007, p. 4) ? Dans le cas des patients conscients et demandant l'arrêt des thérapeutiques, on peut craindre que le consentement du patient, pour demander cet arrêt et accepter le prélèvement, puisse être influencé par des pressions extérieures, en particulier médicales, au moins dans les pays ayant légalisé l'euthanasie. Les soignants eux-mêmes mettent en doute, du fait de leur pratique quotidienne, l'autonomie de patients très dépendants du corps médical, comme on peut l'être dans le cas de pathologies, pour lesquelles la vie est entièrement subordonnée à des techniques de support vital.

Très peu d'études ont, en outre, été réalisées depuis que des prélèvements d'organes ont été entrepris sur des patients conscients après arrêt des traitements. Nous manquons d'informations et d'élaboration analytique réflexive sur les conditions dans lesquelles le consentement a été donné. Certains suggèreront que l'inclusion de ces patients, dans le prélèvement, sous condition du recueil d'un tel consentement, peut s'interpréter en termes d'autonomie des patients concernant leur fin de vie ${ }^{38}$. En effet, on souligne parfois l'existence d'une dissymétrie dans la prise en compte des arguments des uns et des autres qui conduit à ne pas remettre en doute le consentement ni les raisons des patients qui veulent continuer de vivre, dans quelle que condition que ce soit plutôt que celles des patients qui souhaitent mourir (Ogien, 2009, p. 123-125).

\subsection{Abandonner la règle du « donneur mort » ?}

L'un des enjeux moraux et sociétaux cruciaux de l'inclusion des patients DDAC III, dans le prélèvement d'organes, concerne l'abandon de la règle du «donneur mort». Pour les raisons techniques que nous avons précédemment détaillées, le prélèvement sur patients DDAC III pourrait mettre en question cette règle. Elle ouvre l'éventualité d'y renoncer, dans

\footnotetext{
37 Voir la décision du Conseil d'État du 27 octobre 1995, concernant les arrêtés municipaux interdisant les lancers de nains.

${ }^{38} \mathrm{Si}$ pour un patient qui ne serait pas conscient, la question de l'information et du « consentement éclairé » des proches demeure.
} 
ce cas spécifique au moins. Dans cette perspective, doit-on abandonner la règle du « donneur mort » ou y faire une exception pour certains patients? Une telle évolution amorcerait, du point de vue de la législation française actuelle, un «changement de paradigme » (Verheijde et al., 2007) en matière de don d'organes. Serait introduite, en sus de la notion de mort cérébrale, celle de «mort de la conscience » ou de «mort de la vie relationnelle ».

L'abandon de la règle du «donneur mort» reviendrait à autoriser le prélèvement d'organes durant la phase terminale de maladie de patients au pronostic très sévère, après qu'un consentement ait été obtenu. Dans cette perspective, on privilégierait la référence à un «point de non retour », synonyme de déficit neurologique majeur irréversible ou de décès imminent (Nicolas-Robin, 2009, p. 63) ou bien la règle du patient «mourant », s'agissant des patients DDAG III (Verheijde et al., 2007). Ce changement de paradigme ne serait évidemment envisageable qu'à condition que soient mis au point des outils pronostiques, validés internationalement et acceptés par la communauté médicale mondiale. Ces outils devront avoir une spécificité supérieure à $99 \%$ pour prédire l'absence de retour possible à une vie relationnelle. Toutefois leur élaboration n'est pas aujourd'hui achevée.

Cette évolution technologique ne constitue cependant pas, comme telle, une réponse à plusieurs interrogations morales : prélever des organes sur un patient qui va mourir mais qui n'est pas mort, est-ce violer le respect dû à la personne et l'instrumentaliser ? D'un point de vue social et moral - c'est-à-dire pas seulement médical - l'abandon de cette règle est-il acceptable (voir Siminoff et al., 2004)? La condition du consentement explicite ou un choix obligatoire (Verheijde et al., 2007, p. 3), dans ce cas particulier, est-elle suffisante pour protéger le droit des patients à accepter ou à refuser le don d'organes et à justifier l'acceptabilité morale de cette pratique?

D’un point de vue éthique, on sait que les difficultés d'application des normes sont souvent liées à des manières alternatives et également crédibles, à première vue, de qualifier les faits émergents au regard des catégories préexistantes. Sont identifiables des effets propres, suscités par la qualification de nouvelles pratiques, par exemple, de prélèvement d'organes. Il convient de garder présent à l'esprit que les conséquences sociales du choix de la description - ici du prélèvement d'organes sur patients qui ne seraient pas, préalablement, déclarés morts - ne peuvent être négligées ni euphémisées. Incontestablement, ce choix peut être une conséquence d'évolutions spontanées de et dans la société, comme on l'a observé aux PaysBas, en Belgique et dans certains États des États-Unis, ou s'agissant de l'autorisation d'autres pratiques médicales. 
L'abandon de la règle du donneur mort témoignerait alors d'un cas, non pas de réélaboration de normes fondamentales sous l'influence d'une évolution sociale - comme on l'a observé dans les cas de législation de l'euthanasie ou d'assistance médicale à la procréation - mais au contraire d'évolution normative, soutenue par une minorité sociale, imposant à terme un choix sociétal décisif à l'ensemble du corps social. Or cette évolution normative peut être légitimement considérée comme transgressive puisqu'il s'agirait de prélever les organes de personnes qui ne seraient pas déclarées mortes. La profession médicale - et, à terme, la société dans son ensemble - est ici confrontée à un cas majeur de réélaboration de normes fondamentales (et fondatrices de la pratique médicale), indirectement appelée par une demande issue d'un fragment de la société. Cette demande sociale partielle induirait, si elle était acceptée, une reformulation normative fondamentale et une évolution sociale majeure, dont on doit légitimement se demander si elles pourraient moralement être assumées, comme telles, par l'ensemble du corps social. Est-on sûr, dans une telle perspective, de ne pas léser les convictions morales des personnes qui seraient concernées par ces pratiques?

\section{Conclusion}

L'inclusion des patients DDAG III dans le prélèvement d'organes soulève des questions anthropologiques, psychologiques et morales spécifiques. Ces questions mobilisent des représentations culturelles et des imaginaires sociaux caractérisés (Lebreton, 2008, p. 324). L'application des normes et la promotion des valeurs d'un groupe social sont largement tributaires de la perception et de la description des faits concernés (comme pour l'avortement par exemple) mais aussi de la manière dont s'engagent le dialogue social et la délibération, à propos de la réponse à adopter face à une situation donnée. En d'autres termes, la façon dont le débat sera engagé et les termes et descriptions qui seront mobilisés, autour de l'extension des conditions d'éligibilité au prélèvement d'organes, auront un effet décisif sur l'acceptation de cette pratique. La législation d'une telle pratique exprimerait en outre un choix social et une attitude spécifique à l'égard de certains principes moraux, à l'égard des patients sur le point de mourir. Elle reflèterait également un positionnement spécifique face à l'éthique palliative.

L'analyse que nous avons proposée avait pour objectif de systématiser les difficultés, notamment morales, induites par le prélèvement d'organes sur patients DDAG III. Nous avons montré qu'il serait déraisonnable de croire que la légalisation de ce type de prélèvement apporterait une solution - et encore moins, la meilleure solution - au besoin de greffons dans 
le pays. Cette pratique ne doit pas seulement être envisagée quant à ses résultats quantitatifs, en termes de greffons potentiels, mais également par rapport aux coûts qu'elle fera peser sur les équipes, aux dilemmes moraux auxquels elle confrontera les soignants et aux choix sociaux et moraux qu'elle implique pour la société française.

\section{Références}

ARISTOTE, Ethique à Nicomaque, Paris, Vrin, 1990.

BAYLEY C., 2009, «Brain Death », VP Ethics and Justice Education, Catholic Healthcare West, $\quad$ http://www.chwhealth.org/Who_We_Are/Ethics/ssLINK/STGSS044874, consulté le 06/12/09.

BERNAT J. L., 2006a, «Are Organ Donors after Cardiac death Really Dead? », 7 Clin Ethics, vol.17, n², pp.122-132.

BERNAT J. L. et al., 2006b, « Report of a National Conference on Donation after Cardiac Death », American Fournal of Transplantation, vol.6, n² 2, pp.281-291.

BERNAT J. L., 2008, "The Boundaries of Organ Donation after Circulatory Death », New England Fournal of Medecine, vol.359, n², pp.669-671.

BOUCEK, M. M. et al., 2008, «Pediatric Heart Transplantation after Declaration of Cardiocirculatory Death », New England Fournal of Medecine, vol.359, n²7, pp.709-714.

CURFMAN G. D. et al., 2008, "Cardiac Transplantation in Infants », New England fournal of Medecine, vol.359, nº , pp.749-750.

D'ALESSANDRO A. M., J. W. PELTIER et J. E. PHELPS, 2008, « Understanding the antecedents of the acceptance of donation after cardiac death by healthcare professionals ", Critical Care Medicine, vol.36, n 4 , pp.1075-1081.

FISHER C. et al., 2004, "Predictive value of sensory and cognitive evoked potentials for awakening from coma », Neurology, vol.63, nº4, pp.669-673.

FISHER C. et al., 2006, «Improved prediction of awakening or nonawakening from severe anoxic coma using tree-based classification analysis », Crit Care Med, vol.34, n5, pp.15201524.

GUIBET LAFAYE C. et E. PICAVET, 2010, "L'éthique médicale au risque du débat bioéthique et la réponse normative à l'innovation », Revue Philosophique de Louvain, Louvainla-Neuve, p. 687-708.

GUIBET LAFAYE C. et PUYBASSET L., 2010, «Accepter le prélèvement d'organes sur patients DDAC III ? ", dans L. Puybasset (dir.), Enjeux éthiques en réanimation, SpringerVerlag, pp.567-580

HENNETTE-VAUCHEZ S. et G. NOWENSTEIN, 2009, «Dire la mort et faire mourir. Tensions autour de la mort encéphalique et la fin de vie en France », Sociétés contemporaines, 2009/03, n 75, pp.37-57.

LARA M., 2000, "Donor Action en la Comunidad Autónoma de Castilla y León », Communication présentée au $1^{\text {st }}$ Congress of Nephrology in Internet, Burgos, 9 Mars 2000, 
http://www.uninet.edu/cin2000/conferences/MLara/mlaral/index.htm (consulté le 24 mars 2009).

LEBRETON D., 2008, La chair à vif, Paris, Editions Métailié.

NICOLAS-ROBIN A., 2009, «De la neuroréanimation à la réanimation d'organes », Mémoire pour l'obtention du Master «Éthique, science, santé et société », 2ème année à la Faculté de médecine - Université Paris-Sud XI.

OGIEN R., 2009, La vie, la mort, l'État. Le débat bioéthique, Paris, Grasset.

PELLUCHON G., 2009, L'autonomie brisée, Paris, PUF.

RANJAN D. et al., 2006, «Financial analysis of potential donor management at a Medicareapproved transplant hospital », Am J Transplant, vol.6, pp.199-204.

RAWLS J., 1971, Theory of Fustice, Cambridge (Massachusetts), Belknap Press of Harvard University Press ; trad. franç., Théorie de la justice, Paris, Seuil, 1987.

REVELLY J.-P. et al., 2006, « Are terminally ill patients dying in the ICU suitable for nonheart beating organ donation? ", Intensive Care Med, vol.32, pp.708-712.

RIOU B., 2010, « Prélèvement d'organe et Maastricht 3 : la position d'un médecin français », dans L. Puybasset (dir.), Enjeux éthiques en réanimation, Springer-Verlag.

SALIM A. et al., 2007, «Improving consent rates for organ donation: the effect of an inhouse coordinator program », 7 Trauma, vol.62, pp.1411-1415.

SIMINOFF L. A. et al., 2004, «Death and organ procurement: public beliefs and attitudes », Social Science Ë Medicine, vol.59, pp.2325-2334.

SPITAL A., 2003, "Conscription of cadaveric organs for transplantation: neglected again », Kennedy Inst Ethics J, vol.13, pp.169-174.

STEINBROOK R., 2007, "Organ donation after cardiac death », New Eng 7 Med, vol.357, pp.209-213.

STEINER Ph., 2006, "La "productivisation" de la mort: mort encéphalique et transplantation d'organes ", Quaderni, vol.62, n¹, pp.69-80.

TRUOG R.D. et G.M. FRANKLIN, 2008, "The dead donor rule and organ transplantation », New England Fournal of Medecine, vol.359, n 7, pp.674-675.

TUGENDHAT E., 1998, Conférences sur l'éthique, Paris, PUF.

VEATCH R. M., 2008, "Donating Hearts after Cardiac Death - Reversing the Irreversible », New England Fournal of Medecine, vol.359, n 7, pp.672-673.

VERHEIJDE J.L., M. Y. RADY, et J. McGREGOR, 2007, « Recovery of transplantable organs after cardiac or circulatory death: Transforming the paradigm for the ethics of organ donation », Philosophy, Ethics, and Humanities in Medicine, vol.2, n8.

VINCENT J.L. et S. BRIMIOULLE, 2010, « NHBD : aspects éthiques », dans L. Puybasset (dir.), Enjeux éthiques en réanimation, Springer-Verlag.

YSEBAERT D. et al., 2009, "Organ procurement after euthanasia: Belgian experience », Transplant Proc, vol.41, pp.585-586.

ZINGMOND D.S. et N.S. WENGER, 2005, «Regional and institutional variation in the initiation of early do-not-resuscitate orders », Arch Intern Med, vol.165, pp.1705-1712. 